\title{
Research on Radial Motion Characteristic of the Cropping Hammer in Radial-Forging Cropping Method
}

\author{
Lijun Zhang, ${ }^{1}$ Shengdun Zhao, ${ }^{2}$ and Zhenwei Wang ${ }^{2}$ \\ ${ }^{1}$ College of Electromechanical Engineering, China University of Petroleum, Qingdao 266580, China \\ ${ }^{2}$ School of Mechanical Engineering, Xian Jiaotong University, Xian 710049, China \\ Correspondence should be addressed to Lijun Zhang; zlj-2@163.com
}

Received 10 April 2015; Revised 27 May 2015; Accepted 27 May 2015

Academic Editor: Michele Iafisco

Copyright (c) 2015 Lijun Zhang et al. This is an open access article distributed under the Creative Commons Attribution License, which permits unrestricted use, distribution, and reproduction in any medium, provided the original work is properly cited.

\begin{abstract}
The radial loading form applied to the bar is very important for reducing or avoiding the impact and vibration of the radial-forging cropping system and obtaining the high-quality cross section. A new radial stroke loading curve of the cropping hammer based on the cycloid form is proposed and the dynamic model of radial stroke loading mechanism is built. With the aim of obtaining the equivalent stiffness of the bar with $\mathrm{V}$-shaped notch, which is a key parameter affecting the dynamic characteristic of radial stroke loading mechanism, the analytic model of the bar is built and the simulation experiments are designed by means of the orthogonal test method. The analytical results show that the diameter of the bar has the significant influence on the equivalent stiffness of the bar. Furthermore, the equivalent stiffness of the bar with $\mathrm{V}$-shaped notch can be directly calculated according to the equivalent stiffness of smooth bar when $h / d<0.05$ and $r>0.15$. By using the cycloid stroke curve, the cropping experimental results for 45 steel bars and 20 steel bars show that the radial impact and vibration of the cropping system are decreased and the bar cross-section qualities have been significantly improved.
\end{abstract}

\section{Introduction}

The baiting of metal bar is the first process and is widely used to make various parts, such as gear, shaft, and flange. Nowadays, the required baiting quantity is up to tens of millions of tons annually and the demand is still increased year by year [1]. However, in part, the current cropping processes are plagued with a big shearing force, a bad crosssection quality, and a long cropping time. Therefore, a newtype method with the fatigue radial-forging cropping is proposed [2]. The method is a promising low-stress blanking. In course of cropping, the machine skillfully utilizes the fatigue radial-forging action to make the crack propagate along the prefabricated V-shaped notch in metal bar for shearing bar, and it is also a fast and energy-saving cropping system because of stress concentration effect of V-shaped notch and vibration action [3].

However, the experimental results show that the radial impact and vibration of the cropping system are very big, and the bar is often stuck in cropping die to affect the cross-section quality and cropping efficiency. After repeated analysis, it is found that these problems are closely related with the working profile of the hammer mechanism. Tong et al. designed a cropping hammer and did not study its working characteristic [4]. Li and Zhou analyzed the effect of hammer on the forging penetration efficiency in radial forging and pointed out that using the hammer with performing section is helpful to improve the forging penetration efficiency of forgings [5]. Li et al. designed the hammer of crusher and pointed out that hammer form not only affects the collision balance but also prolongs its working life [6]. Yan and Gong studied the application of different shape rammers in construction of lime-soil compaction piles [7]. However, none of these studies are related to the characteristic analysis of the cropping hammer in precision cropping.

Therefore, the new radial stroke loading curve based on cycloid form is proposed. The dynamics model of the radial stroke loading mechanism is established. Based on it, the working profile model of the cropping hammer is obtained and its stress analysis is also carried out. The cropping experimental results confirm the effectiveness of cycloid stroke curve. 


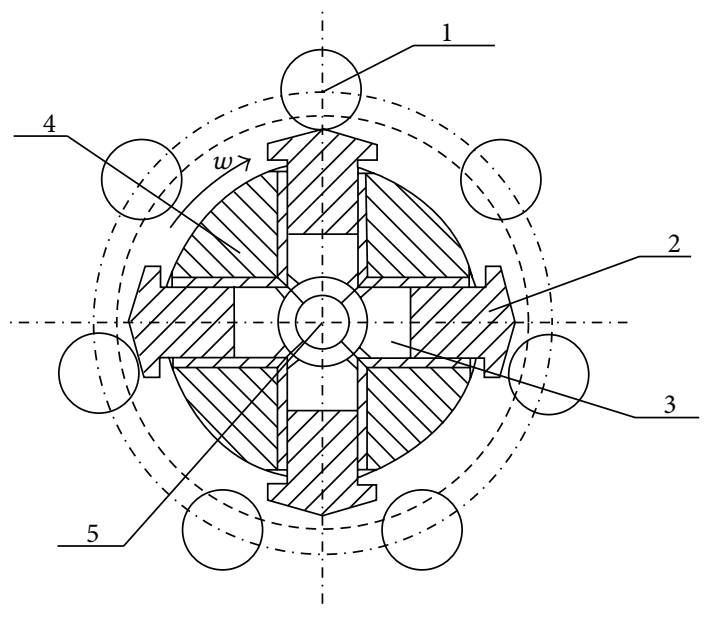
(1) Feeding cylinder roller
(4) Main shaft
(2) Cropping hammer
(5) Metal bar
(3) Blanking die

FIGURE 1: The work principle of fatigue radial-forging cropping.

\section{The Work Principle of the Fatigue Radial-Forging Cropping}

The work principle of fatigue radial-forging cropping is shown in Figure 1. The radial stroke loading mechanism of the cropping machine consists of feeding cylinder roller, cropping hammer, blanking die, and main shaft. Before the cropping machine works, one end of the bar with V-shaped notch is fixed and another end is placed at the center of main shaft. In the plane perpendicular to the axis of the bar, there are seven stationary feeding cylinder rollers and four cropping hammers. These hammers are homogeneously inlaid in guide chute of main shaft and can rotate with the main shaft synchronously.

In the cropping, only a cropping hammer is in contact with a feeding cylinder roller at a time, which can make the bar in the radial load state. The motions among the cropping hammer do not interfere with each other. Thus the load acted on the bar is distributed uniformly along the bar circumference. When the main shaft rotates one circle, the bar can be uniformly hit many times. Because the speed of main shaft is up to $3000 \mathrm{r} / \mathrm{min}$, the crack of $\mathrm{V}$-shaped notch tip can be uniformly expanded until the bar fractures.

\section{Radial Stroke Curve of the Cropping Hammer}

3.1. Analysis of Original Radial Stroke Curve of the Cropping Hammer. According to the work principle of radial-forging cropping, the radial stroke loading is carried out by contact of the feeding cylinder roller and the cropping hammer. Therefore, the working profiles of feeding cylinder roller and the cropping hammer decide the loading form jointly. The exterior outline of feeding cylinder roller is a regular circle and only the working profile of the cropping hammer needs to be designed. The radial accelerations of cropping hammer in the case of original cosine and sine stroke curve are shown in Figure 2. As shown in Figure 2, for the cosine stroke curve, when the feeding cylinder roller and the cropping hammer begin to contact, namely, the movement angle is equal to zero, the radial acceleration of cropping hammer reaches the maximum value, which leads to big radial inertial force to cause the radial impact and vibration.

For the sine stroke curve, when the feeding cylinder roller and the cropping hammer begin to contact, the acceleration curve of cropping hammer is not continuous and has an inflection point, which can also cause the radial impact and vibration of the cropping hammer.

3.2. Design of New Radial Stroke Curve of the Cropping Hammer. The basic design principle of working profile of the cropping hammer is to ensure the continuity and stability of radial stroke loading mechanism motion in the fatigue radial-forging cropping and to reduce or avoid the excessive vibration and impact. Based on it, the new radial stroke loading curve based on cycloid form is proposed and the expression of cycloid stroke curve is given by

$$
\begin{aligned}
& s(a) \\
& = \begin{cases}h_{0}\left(\frac{a}{a_{0}}-\frac{1}{2 \pi} \sin \frac{2 \pi a}{a_{0}}\right) & {\left[0 \leq a \leq a_{0}\right]} \\
h_{0}\left(\frac{2 a_{0}-a}{a_{0}}-\frac{1}{2 \pi} \sin \frac{2 \pi\left(2 a_{0}-a\right)}{a_{0}}\right) & {\left[a_{0} \leq a \leq 2 a_{0}\right],}\end{cases}
\end{aligned}
$$

where $s(a)$ is the radial stroke of the cropping hammer and it is a function of movement angle $a$. The first equation in (1) is a lifting curve and the second equation is a returning curve. The whole cycloid stroke curve is shown in Figure 3, which is smooth, continuous, and symmetric.

\section{The Dynamic Characteristic of Radial Stroke Loading Mechanism}

4.1. Analysis of the Generalized Force Applied on the Bar. To verify the effectiveness of cycloid stroke curve, the radial stroke loading mechanism is regarded as a research object. The global coordinate system $x-y$, local coordinate system $x_{i}-y_{i}$, and $x_{j}-y_{j}$ are established and the dynamic simplified model of the radial stroke loading mechanism is shown in Figure 4 . The force $F_{P}$ of feeding cylinder roller is along the normal direction of cropping hammer. Because the relative motion between the feeding cylinder roller and the cropping hammer is pure rolling, the friction force between them is negligible. The action of main shaft on the cropping hammer is simplified as the concentrated force $F_{C}$ and torque $M_{C}$ in position of cropping hammer centroid. In addition, the bottom of the cropping hammer is directly in contact with the sleeve, and the displacement of contact point along $x$ axis is smaller, which is regarded as $F_{i x}=0$. Based on it, 


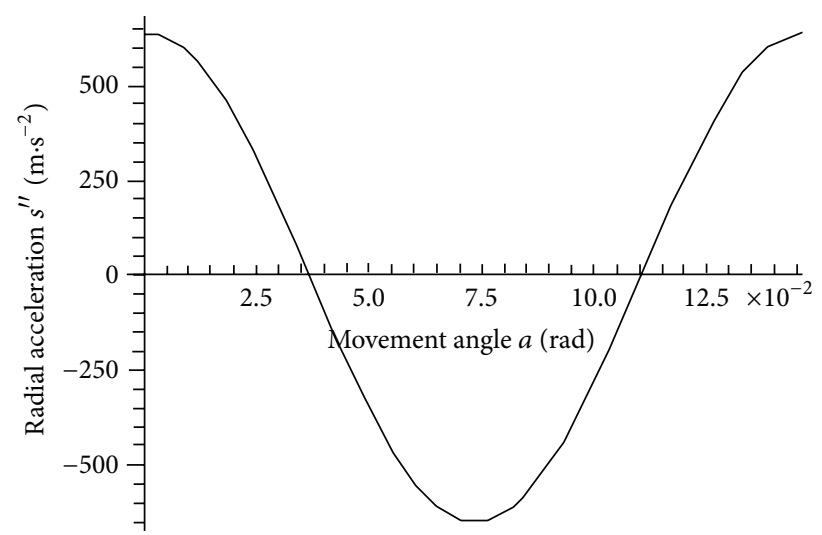

(a) Cosine stroke curve

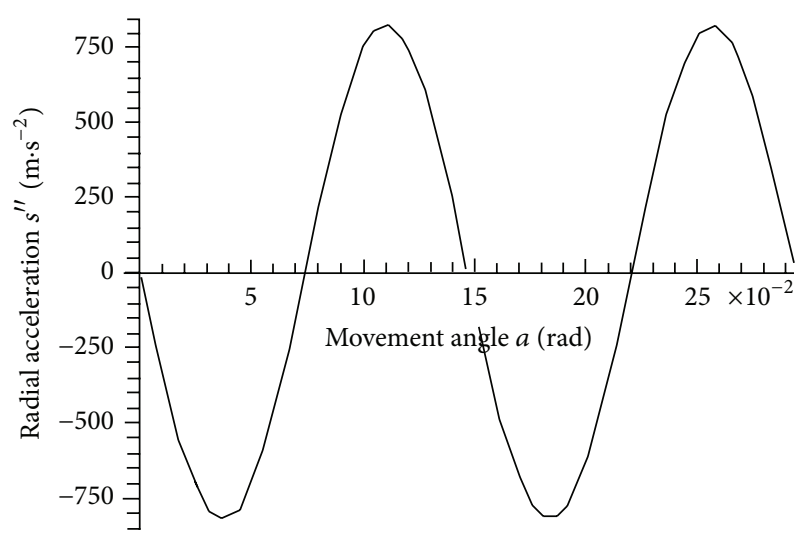

(b) Sine stroke curve

FIGURE 2: The radial acceleration curve of cropping hammer in different stroke curve.

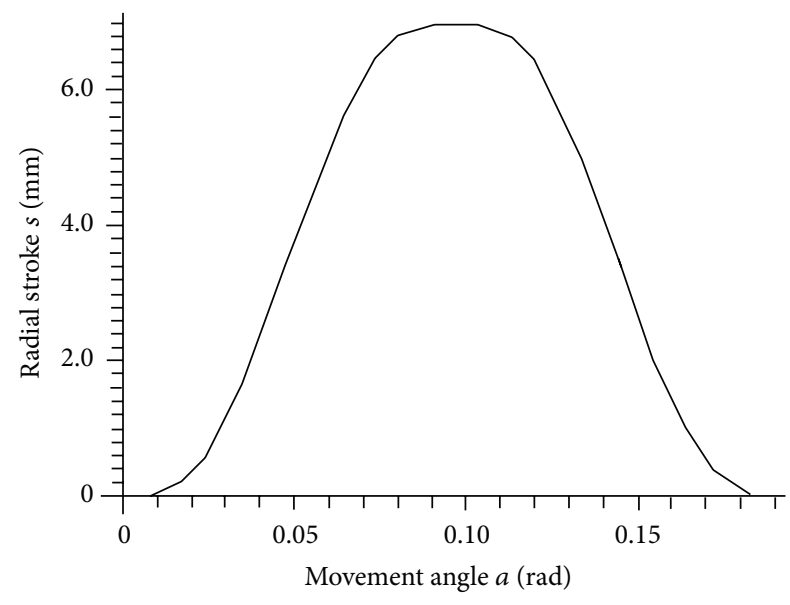

FIGURE 3: The whole cycloid stroke curve.

the generalized force vector of cropping hammer is expressed as $\mathbf{Q}=\left[\begin{array}{llll}F_{C} & F_{i y} & F_{P} & M_{C}\end{array}\right]^{T}$. According to kinetic law [8], the expression of inertia force of the cropping hammer is expressed as [2]

$$
\mathbf{K}_{4 \times 4} \cdot \mathbf{Q}=\mathbf{N} .
$$

Based on the dynamics principle and the design parameters of cropping mechanism, the coefficient matrix of the radial stroke loading mechanism is given by

$$
\mathbf{K}=\left[\begin{array}{cccc}
\cos \phi_{i} & -\sin \phi_{i} & -\sin a_{p} & 0 \\
\sin \phi_{i} & \cos \phi_{i} & -\cos a_{p} & 0 \\
0 & 0 & -l_{p} & 1 \\
0 & 1 & 0 & 0
\end{array}\right] .
$$

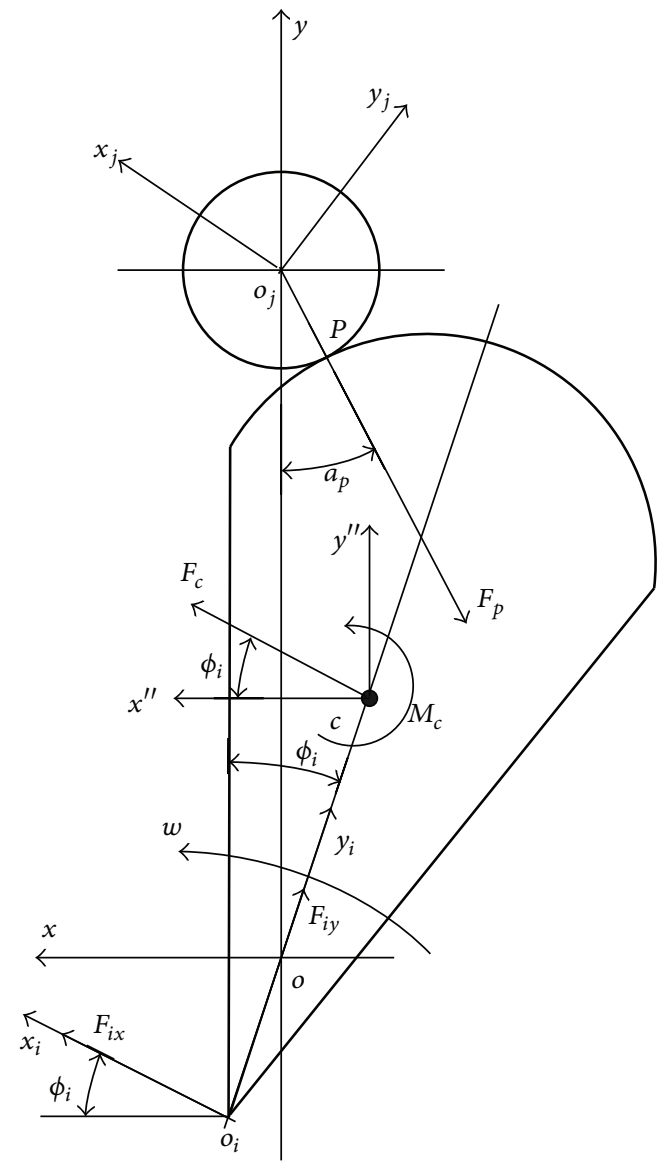

FIGURE 4: The dynamic simplified model of the radial stroke loading mechanism.

When the coefficient matrix is nonsingular, namely, $|\mathbf{K}| \neq$ 0 , then

$$
\mathbf{Q}=\mathbf{K}^{-1} \cdot \mathbf{N}
$$


Based on it, the generalized force applied on the bar can be obtained by

$$
\begin{aligned}
\mathbf{Q} & =\left[\begin{array}{c}
F_{C} \\
F_{i y} \\
F_{p} \\
M_{c}
\end{array}\right] \\
& =\left[\begin{array}{cccc}
\cos \phi_{i} & -\sin \phi_{i} & -\sin a_{P} & 0 \\
\sin \phi_{i} & \cos \phi_{i} & -\cos a_{P} & 0 \\
0 & 0 & -l_{P} & 1 \\
0 & 1 & 0 & 0
\end{array}\right]^{-1}\left[\begin{array}{c}
m \ddot{x}_{i} \\
m \ddot{y}_{i} \\
J w \\
-k y_{i}
\end{array}\right] .
\end{aligned}
$$

In order to solve the above generalized force $\mathbf{Q}, l_{p}, k$, and $\mathbf{r}_{c}^{\prime}=\left[\begin{array}{ll}x_{c}^{\prime} & y_{c}^{\prime}\end{array}\right]^{T}$ need to be determined. According to the design structure of the cropping hammer, its centroid coordinate can be expressed as

$$
\begin{aligned}
& x_{c}^{\prime}=0 \\
& y_{c}^{\prime}=\frac{\iint_{\Omega} y \cdot \rho d x d y}{m}=109.64 \mathrm{~mm} \approx 110 \mathrm{~mm} .
\end{aligned}
$$
by

The centroid vector of the cropping hammer is obtained

$$
\mathbf{r}_{c}=\left[\begin{array}{c}
x_{i}-\sin \phi_{i} \cdot\left|\mathbf{r}_{c}^{\prime}\right| \\
y_{i}+\cos \phi_{i} \cdot\left|\mathbf{r}_{c}^{\prime}\right|
\end{array}\right] .
$$

According to the geometrical relationship as shown in Figure $4, l_{p}$ is expressed as

$$
l_{p}=223 \sin a_{P}-\left|\mathbf{r}_{c}\right| \sin \left(a_{P}+\phi_{i}\right) .
$$

According to the known conditions $\phi_{i}=a_{0}-a$, the changing curve of $l_{p}$ can be obtained and shown in Figure 5. In addition, according to material mechanics and the simulation model of metal bar as shown in Figure 6, the flexibility $f$ of the bar cantilever end in the action of $F_{i y}$ is expressed as

$$
f=\frac{F_{i y} l^{3}}{3 E I} .
$$

Thus the equivalent stiffness of the bar is written as

$$
k=\frac{F_{i y}}{f}=\frac{3 E I}{l^{3}} .
$$

When $m=4.3 \mathrm{~kg}$ and $J=12.3 \times 10^{-3} \mathrm{~kg} \cdot \mathrm{m}^{2}$, the equivalent stiffness of the bar is equal to $3.54 \times 10^{7} \mathrm{~N} \cdot \mathrm{m}^{-1}$.

Substituting (8) and (10) into (5), the changing curves of the main generalized force components are shown in Figure 7. As shown in Figure 7, these curves are very smooth within the movement angle, which verifies the effectiveness of the cycloid stroke curve.

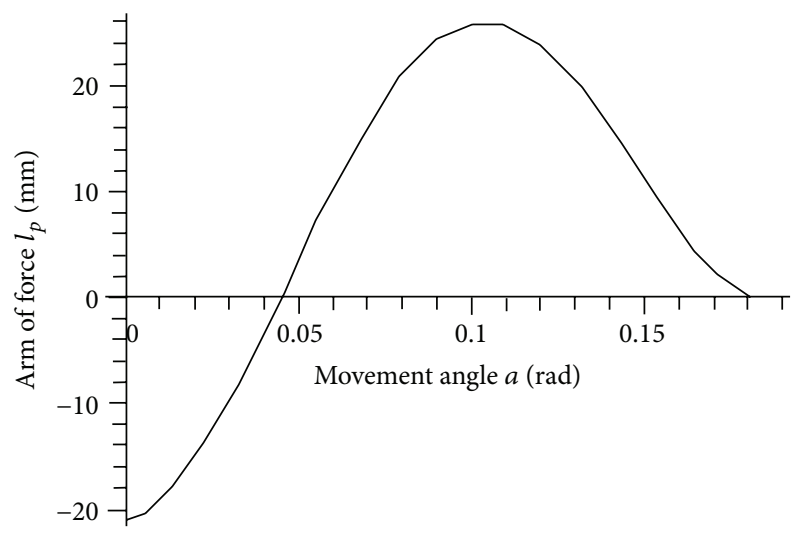

FIGURE 5: The changing curve of the arm of force.

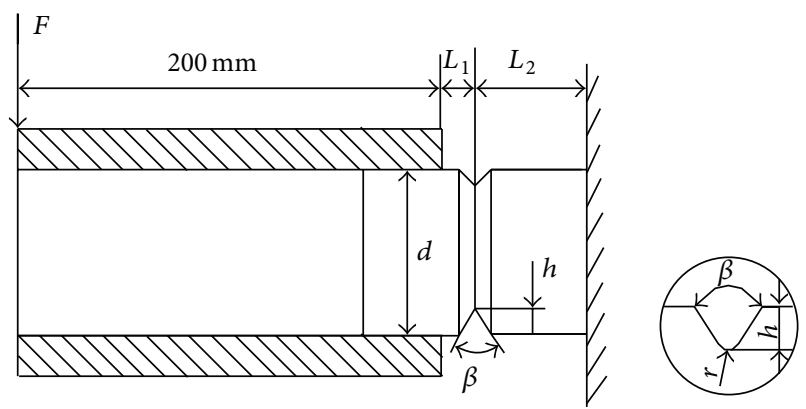

Figure 6: The finite element analysis model of the bar.

4.2. Determination of Cropping Hammer Structure. The coefficient matrix $\mathbf{K}$ in (2) is calculated as

$$
|\mathbf{K}|=-\cos \left(\phi_{i}+a_{p}\right) .
$$

According to the movement of cropping hammer, the following relation can be obtained:

$$
\phi_{i}+a_{P}<\frac{\pi}{2}
$$

Substituting (12) into (11), the coefficient matrix is calculated as

$$
|\mathbf{K}|=-\cos \left(\phi_{i}+a_{p}\right)<0 \Longrightarrow|\mathbf{K}| \neq 0 .
$$

Therefore, (4) meets the conditions of solvability and has a unique solution. In addition, there is no connection between $|\mathbf{K}|$ and $l_{p}$; namely, the contact movement configuration between the cropping hammer and the feeding cylinder roller is without isomerism. By the above analysis, the cycloid stroke curve is feasible and the model of the cropping hammer is obtained in Figure 8.

4.3. Analyses of Stress and Strain of the Cropping Hammer. In order to verify the strength and stiffness of the cropping hammer, the finite element analysis is carried out. The analysis results of equivalent stress and equivalent strain cloud of the cropping hammer are shown in Figure 9. As is shown in Figure 9, the maximum equivalent stress, $396.5 \mathrm{MPa}$, and the 

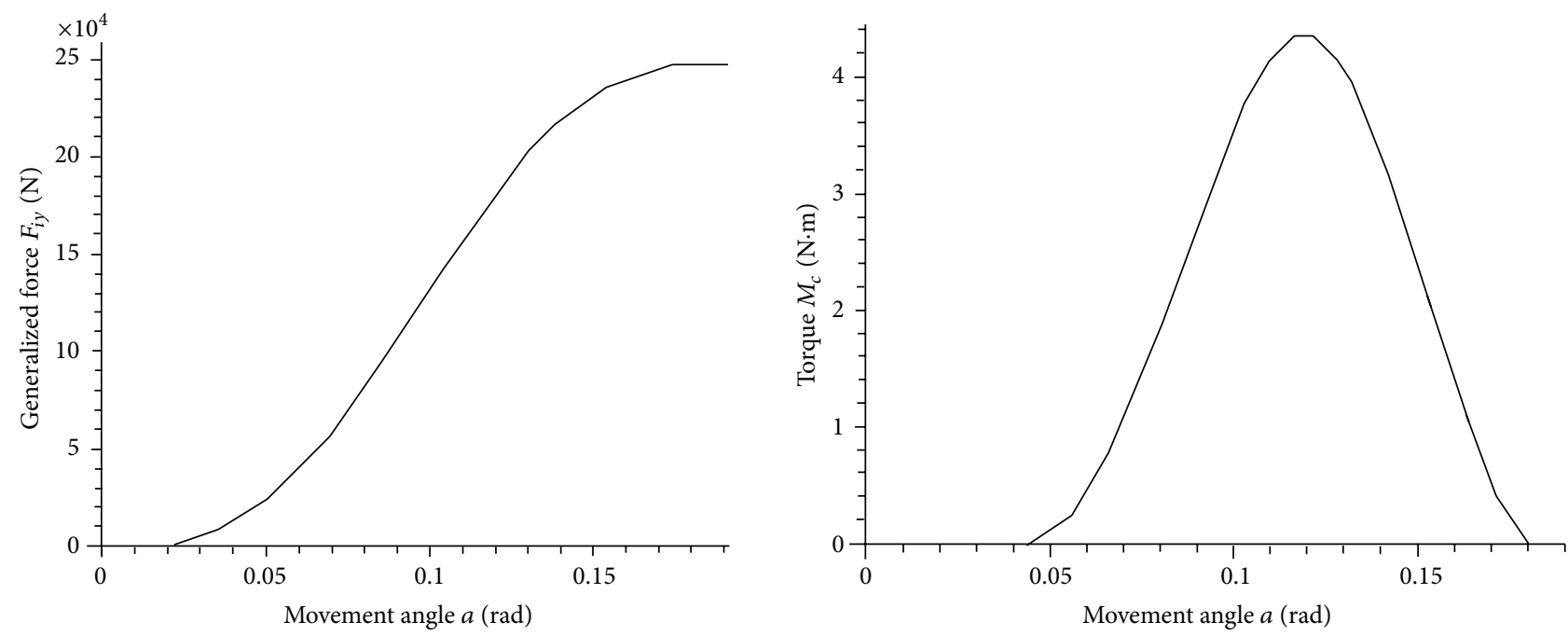

FIgURE 7: The changing curves of the main generalized force components.

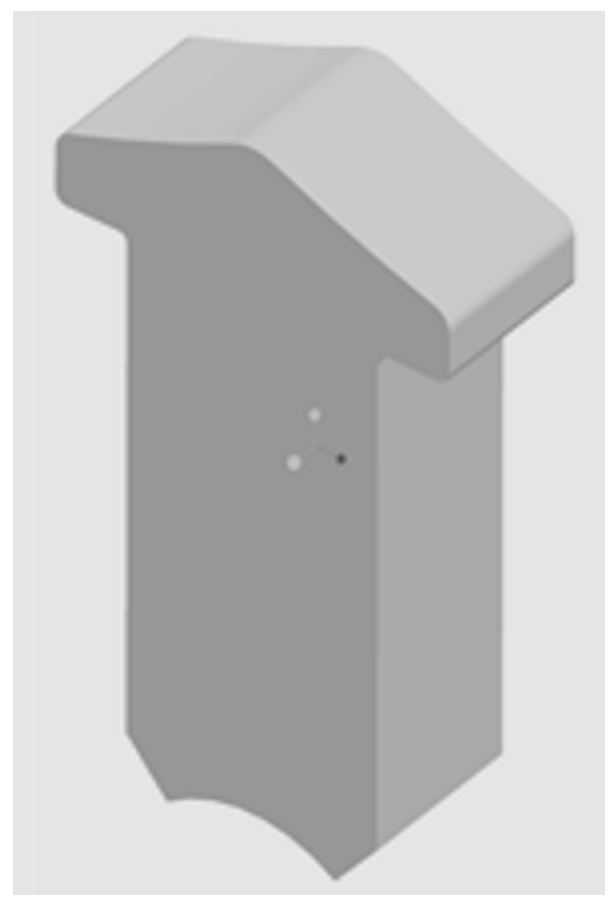

FIGURE 8: The model of the cropping hammer.

maximum equivalent strain, 0.00198 , are located in the contact zone between the feeding cylinder roller and the cropping hammer. The material of the cropping hammer is $40 \mathrm{Cr}$, and its yield strength is $490 \mathrm{MPa}$; hence the cropping hammer can meet the requirement of material strength.

4.4. Analysis of Equivalent Stiffness of the Bar. Equation (4) shows that the equivalent stiffness of the bar has a direct influence on the dynamic characteristic of the radial stroke loading mechanism. However, the equivalent stiffness of the bar changes with the changing of the bar geometry parameters. It is very difficult to calculate the equivalent stiffness of the bar with V-shaped notch by using the analytic method [9]. In order to analyze the influence of bar parameters on the equivalent stiffness of the bar, the orthogonal test method is applied in this paper. The 6 factors, which are flare angle $\beta$, groove depth $h$, diameter $d$ of the bar, distance $L_{1}$ between the left end of the sleeve and V-shaped notch, distance $L_{2}$ between the clamping position and V-shaped notch, and radius $r$ at the groove bottom, respectively, are set. Every factor selects 5 levels. The specific value of every parameter is shown in Table 1.

Because the equivalent stiffness of the bar can be determined by the deformation of the bar cantilever end in (11), the deformation of the bar cantilever end obtained by finite element analysis is regarded as the analytical object to study the equivalent stiffness of the bar. In order to further analyze the influence of bar parameters on the equivalent stiffness, the finite element analysis model of the bar is shown in Figure 6. The analysis of variance method based on the orthogonal test is used and the analytic results are shown in Table 2. As shown in Table 2, the influence of diameter of the bar is maximal and its significant level is one time bigger than the significant level of radius at the groove bottom. By repeated analysis, it is obtained that the equivalent stiffness of the bar with $\mathrm{V}$ shaped notch can be calculated by the equivalent stiffness of the smooth bar when $h / d<0.05$ and $r>0.15$.

4.5. Experimental Results and Discussion. Photo of the radialforging cropping system based on the cropping hammer with the cycloid form is shown in Figure 10, and the experimental results for 45 steel bars and 20 steel bars show that the radial impact and vibration of the cropping system are very small.

More importantly, according to the signal data of the acceleration sensor installed on the blanking die, the probability of bar instantaneous fracture caused by the excessive vibration of radial stroke loading mechanism is greatly 


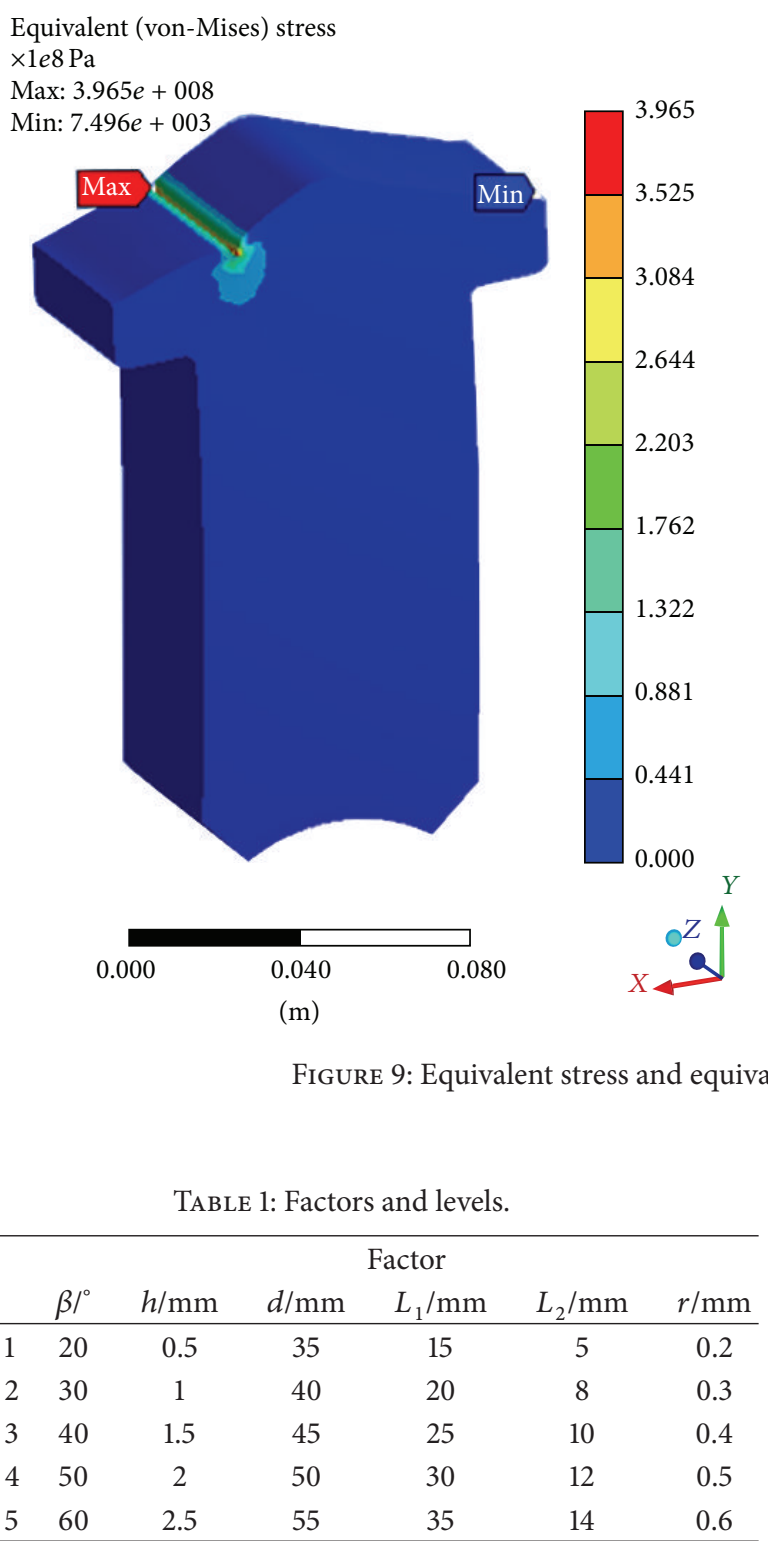

reduced and the cross-section qualities have been significantly improved as shown in Figure 11. As shown in Figure 11, the transient fault zone in bar cross section is almost out of sight by using cycloid stroke loading curve.

\section{Conclusions}

(1) According to the work principle of fatigue radialforging cropping, it is pointed out that the working profile of the cropping hammer has an important influence on the radial vibration of the cropping system, the cross-section quality, and the cropping efficiency.

(2) The radial stroke loading curve of the cropping hammer with cycloid form is proposed. The analysis results of stress and strain of the cropping hammer are obtained. The dynamic analysis of radial stroke loading mechanism shows that the cycloid stroke curve
Equivalent (von-Mises) elastic strain

$\times 1 e-2 \mathrm{~m} / \mathrm{m}$

Max: $1.983 e-003$

Min: $3.748 e-008 \square$
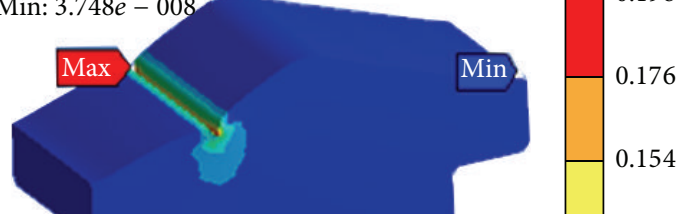

0.132
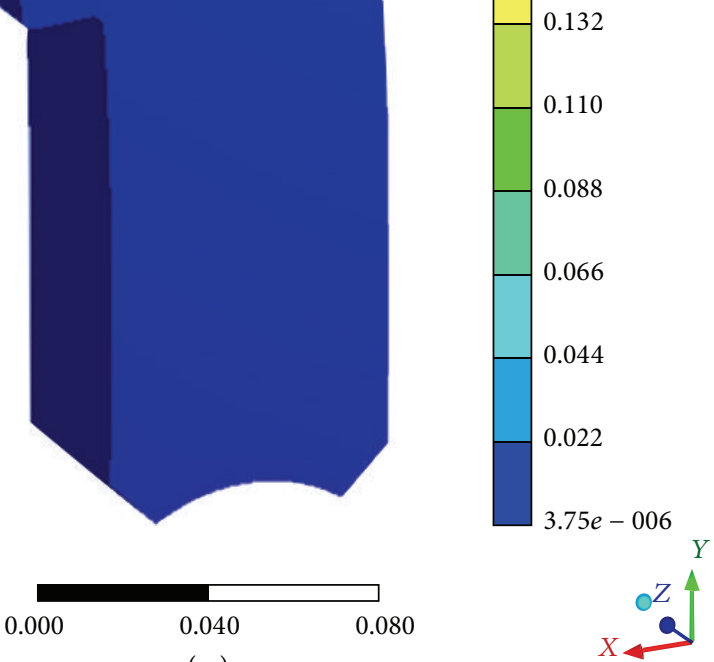

(m)

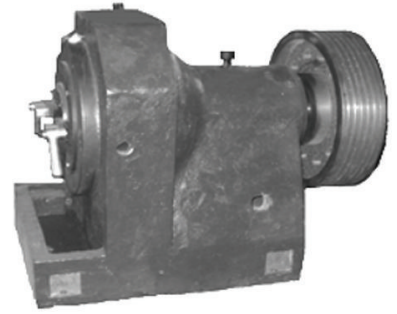

Feeding cylinder roller

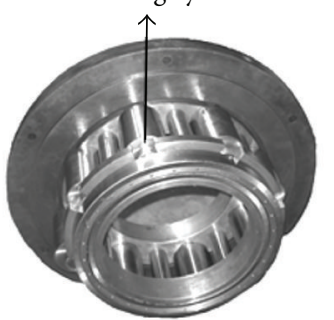

FIGURE 10: Photo of radial-forging cropping system.

can ensure the smooth and continuity of motion characteristic of cropping hammer. Based on it, the structure of the cropping hammer is obtained and used in the radial-forging cropping machine.

(3) The analytical results based on variance analysis show that the bar diameter has the most significant influence on the equivalent stiffness of the bar. 
TABLE 2: The results of analysis of variance.

\begin{tabular}{lcccc}
\hline Factor & Deviation sum of squares & Degree of freedom & $F$ & $F$ critical value \\
\hline$\beta$ & 2.553 & 4 & 1.000 & 6.390 \\
$h$ & 2.958 & 4 & 1.159 & 6.390 \\
$L_{1}$ & 7.344 & 4 & 2.877 & 6.390 \\
$L_{2}$ & 12.141 & 4 & 4.756 & 6.390 \\
$d$ & 43.532 & 4 & 17.051 & 6.390 \\
$r$ & 19.815 & 4 & 7.761 & 6.390 \\
Error & 2.55 & 4 & & \\
\hline
\end{tabular}

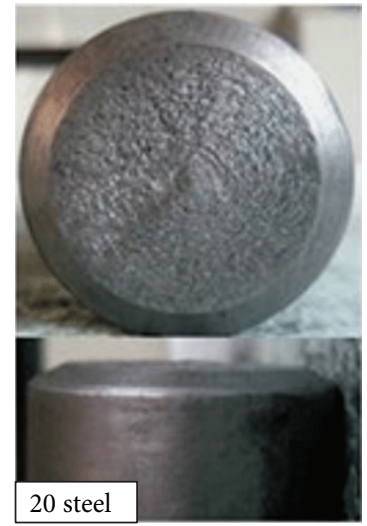

(a) Cycloid stroke curve

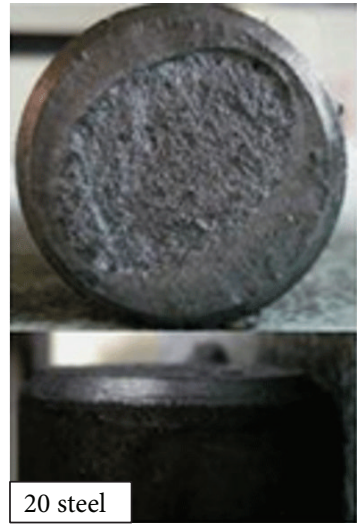

(b) Cosine stroke curve

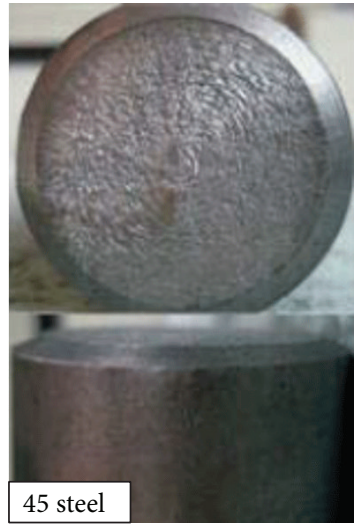

(c) Cycloid stroke curve

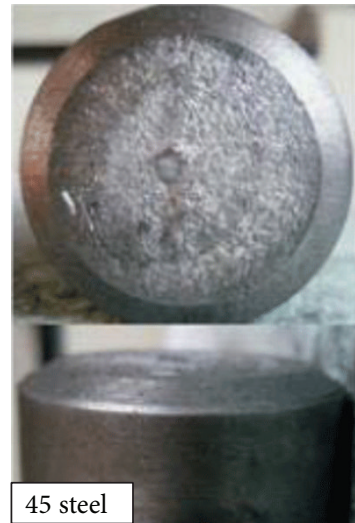

(d) Cosine stroke curve

FIGURE 11: The cross sections for 20 steel bars and 45 steel bars in different stroke curve.

The equivalent stiffness of the bar with V-shaped notch can be directly calculated according to the equivalent stiffness of smooth bar when $h / d<0.05$ and $r>0.15$, which provides an important reference to fast calculate the equivalent stiffness of the bar with $\mathrm{V}$-shaped notch in the radial-forging cropping or in other industry applications.

(4) By using the new cropping hammer with the cycloid stroke curve, the cropping experimental results for 45 steel bars and 20 steel bars show that the radial impact and vibration of the cropping system have been greatly reduced and the cross-section quality of the bar has also been significantly improved.

\section{Nomenclature}

$s(a): \quad$ Radial stroke of the cropping hammer

$a$ and $a_{0}$ : The movement angle of the cropping hammer and its maximum angle

$h_{0}$ : The maximum radial stroke of the cropping hammer, designed as $7 \mathrm{~mm}$

$F_{P}: \quad \quad$ Force of feeding cylinder roller

$F_{C}$ and $M_{C}$ : Concentrated force and torque in position of the cropping hammer centroid

$F_{i x}$ and $F_{i y}$ : Contact forces along $x$-, $y$-axis

$\mathbf{K}_{4 \times 4}: \quad$ Coefficient matrix of the cropping hammer, also called $\mathbf{K}$
Q: $\quad$ Generalized force vector

$\mathbf{N}$ : Inertia force of the cropping hammer, $\mathbf{N}=\left[\begin{array}{llll}m \ddot{x} & m \ddot{y} & J w & -k y\end{array}\right]^{T}$

$m: \quad$ Mass of the cropping hammer

$J$ : $\quad$ Moment of inertia of the cropping

hammer around its centroid

$w: \quad$ Angular frequency of the cropping hammer

$\ddot{x}$ and $\ddot{y}$ : Accelerations of the cropping hammer centroid along $x-, y$-axis

$k: \quad$ Equivalent stiffness of the bar

$x$ and $y$ : Position components of the cropping hammer centroid along $x$-, $y$-axis

$x_{i}, y_{i}: \quad$ Local coordinate system

$\phi_{i}$ : $\quad$ Movement angle of the cropping hammer

$a_{p}: \quad$ Contact angle of the cropping hammer

$l_{p}$ : $\quad$ Length of arm of force between the cropping hammer and the feeding cylinder roller

E: $\quad$ Modulus of elasticity of bar material

I: $\quad$ Moment of inertia of bar cross section, $I_{x}=I_{y}=\pi d^{4} / 64$

$f: \quad$ The flexibility of the bar

$l: \quad$ Cantilever length of the bar

$L_{1}$ and $L_{2}$ : Distance between the left end of the sleeve and V-shaped notch and distance between the clamping position and V-shaped notch

$d$ : $\quad$ Diameter of the bar 
$\beta, h$, and $r$ : Three geometrical parameters of $\mathrm{V}$-shaped notch, see in Figure 6

$x_{c}^{\prime}, y_{c}^{\prime}$ : Centroid coordinate of the cropping hammer.

\section{Conflict of Interests}

The authors declare that there is no conflict of interests regarding the publication of this paper.

\section{Acknowledgments}

The authors wish to express gratitude to the National Natural Science Foundation of China (Approval no. 51105382) and Shandong Natural Science Foundation of China (Approval no. ZR 2011EL039), which have supported this work.

\section{References}

[1] X. M. Sheng and C. H. Deng, Advanced Manufacturing Technology, Machinery Industry Press, Beijing, China, 2011.

[2] Z. W. Wang, Study on the new type radial-forging fatigue cropping method of metal bar and its fatigue and fracture rules [Ph.D. thesis], Xian Jiaotong University, Xi'an, China, 2008.

[3] L. Zhang, S. Zhao, and Z. Wang, "Investigation on the crack initiation of V-shaped notch tip in precision cropping," Advances in Materials Science and Engineering, vol. 2014, Article ID 861465, 8 pages, 2014.

[4] Y. Tong, S. D. Zhao, and Z. W. Wang, "Development of a new type precision cropping machine with rotary striking," CMET Metal forming Equipment and Production Technology, no. 6, pp. 14-17, 2010.

[5] H. Li and X. D. Zhou, "Effect of hammer on forging penetration efficiency in radial forging," Hot Working Technology, vol. 43, no. 5, pp. 138-141, 2014.

[6] Z. F. Li, Y. Su, and L. Q. Jiang, "Improved design of PC600 $\times 400$ hammer crusher hammer," Coal Mine Machinery, vol. 35, no. 11, pp. 201-202, 2014.

[7] F. X. Yan and T. Z. Gong, "Study on applications of different shape rammers in construction of lime-soil compaction piles," Geotechnical Investigation \& Surveying, no. 4, pp. 26-29, 2014.

[8] Y. P. Cheng, Theoretical Mechanics, Harbin Institute of Technology Press, Harbin, China, 2008.

[9] J.-L. Lou and J.-Y. Tang, "A method of equivalent stiffness identification for bearing joint of a gearbox based on CAE/CAT/CAO," Journal of Vibration and Shock, vol. 33, no. 23, pp. 81-86, 2014. 

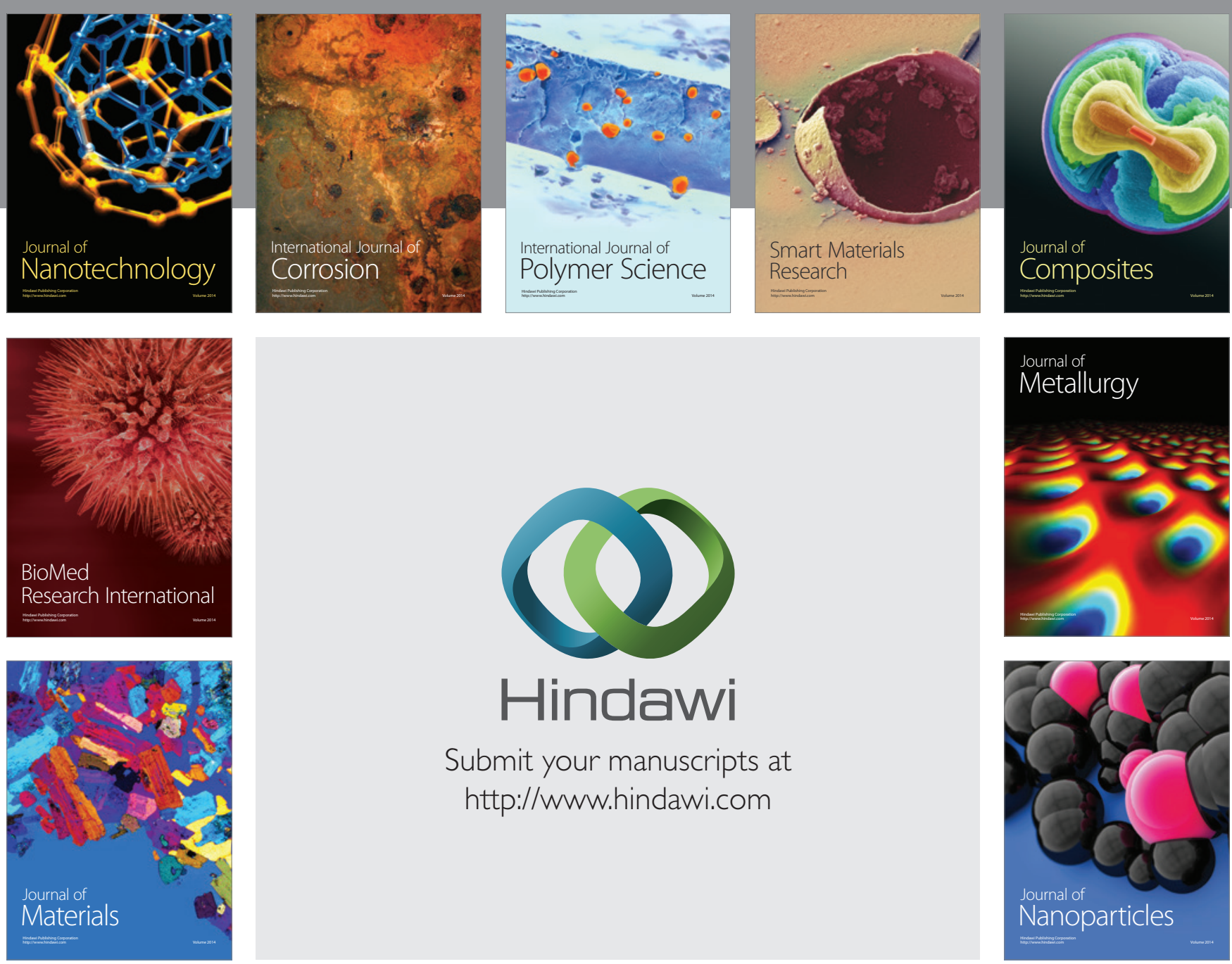

Submit your manuscripts at http://www.hindawi.com
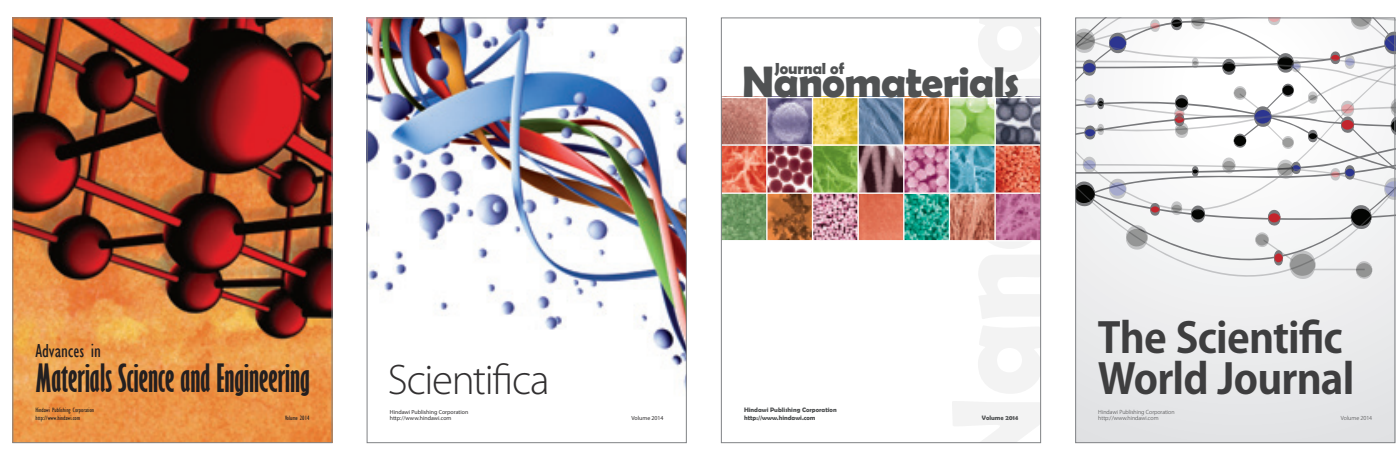

\section{The Scientific World Journal}
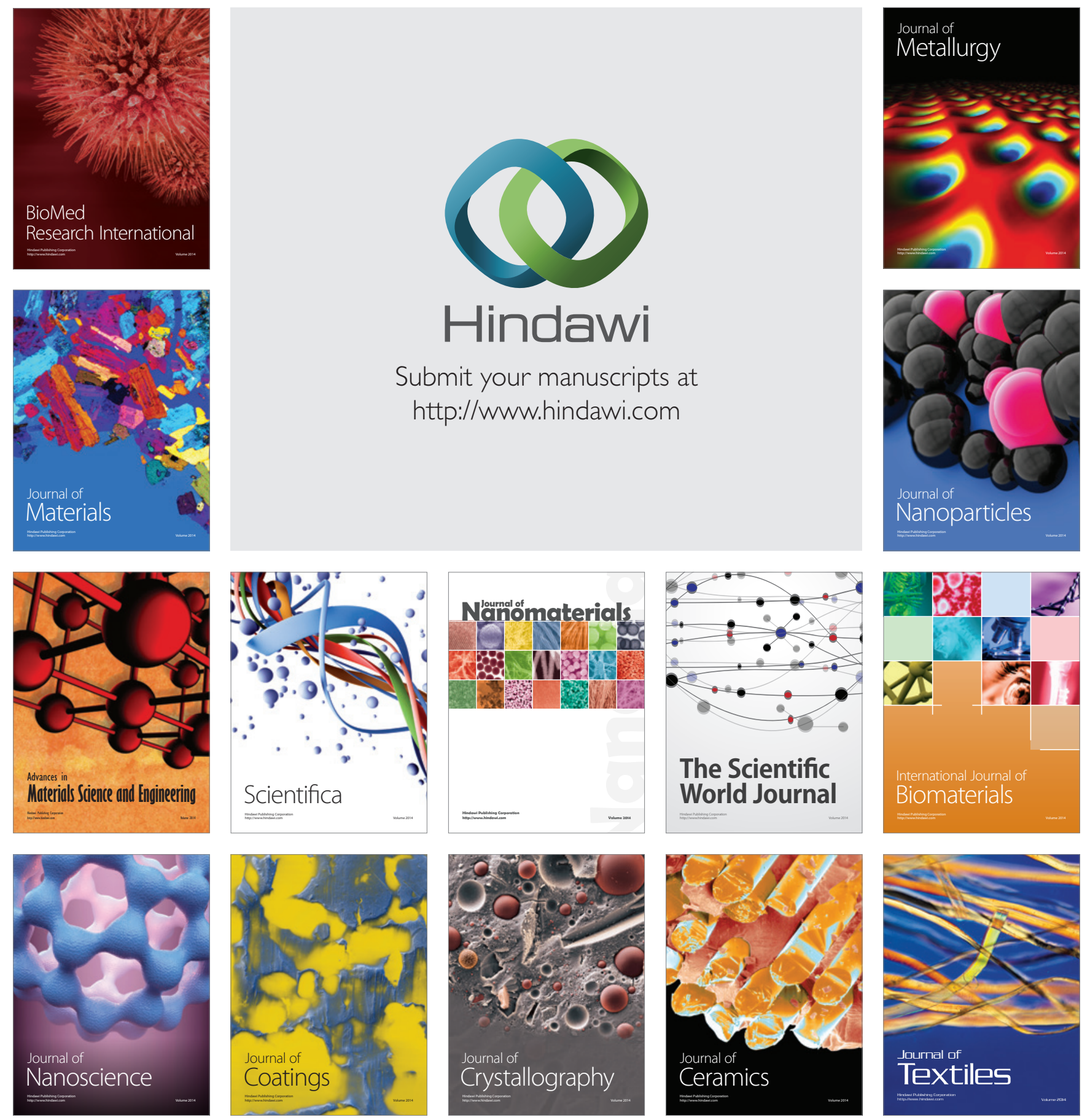